\title{
Nonvariceal upper gastrointestinal hemorrhage: European Society of Gastrointestinal Endoscopy (ESGE) Cascade Guideline
}

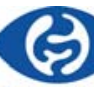

ESGE

\section{(c) (1) $(9)$}

Authors

John Gásdal Karstensen ${ }^{1,2}$, Alanna Ebigbo ${ }^{3}$, Lars Aabakken ${ }^{4}$, Mario Dinis-Ribeiro $^{5}$, Ian Gralnek ${ }^{6}$, Olivier Le Moine ${ }^{7}$, Peter Vilmann ${ }^{1}$, Uchenna Ijoma ${ }^{8}$, Gideon Anigbo ${ }^{9}$, Mary Afihene ${ }^{10}$, Babatunde Duduyemi ${ }^{11}$, Thierry Ponchon ${ }^{12}$, Cesare Hassan $^{13}$

Institutions

1 Gastro Unit, Division of Endoscopy, Copenhagen University Hospital Herlev, Copenhagen, Denmark

2 Gastro Unit, Division of Surgery, Copenhagen University Hospital Hvidovre, Copenhagen, Denmark

3 Department of Gastroenterology, Klinikum Augsburg, Augsburg, Germany

4 Endoscopy, Oslo University Hospital - Rikshospitalet, Oslo, Norway

5 CIDES/CINTESIS, Faculty of Medicine, University of Porto, Porto, Portugal

6 GI Outcomes Unit, Department of Gastroenterology, Rambam Health Care Campus, Rappaport Faculty of Medicine, Technion-Israel Institute of Technology, Haifa, Israel

7 Gastroenterology Department, Erasme University Hospital, Brussels, Belgium

8 Gastroenterology Unit, Department of Medicine, University of Nigeria Teaching Hospital, Ituku-Ozala, Nigeria

9 Department of Medicine, Enugu State University Teaching Hospital, Enugu, Nigeria
10 Department of Medicine, Kwame Nkrumah University of Science and Technology, Kumasi, Ghana

11 Department of Pathology, Kwame Nkrumah University of Science and Technology, Kumasi, Ghana

12 Gastroenterology and Endoscopy, Edouard Herriot Hospital, Lyon, France

13 Endoscopy Unit, Nuovo Regina Margherita Hospital, Rome, Italy

Bibliography DOI https://doi.org/10.1055/a-0677-2084 |

Endoscopy International Open 2018; 06: E1256-E1263

(c) Georg Thieme Verlag KG Stuttgart · New York

ISSN 2364-3722

Corresponding author

Cesare Hassan, MD, Endoscopy Unit, Nuovo Regina

Margherita Hospital, Via Morosini 30, Rome 00153, Italy

Fax: +39-06-58446533

cesareh@hotmail.com

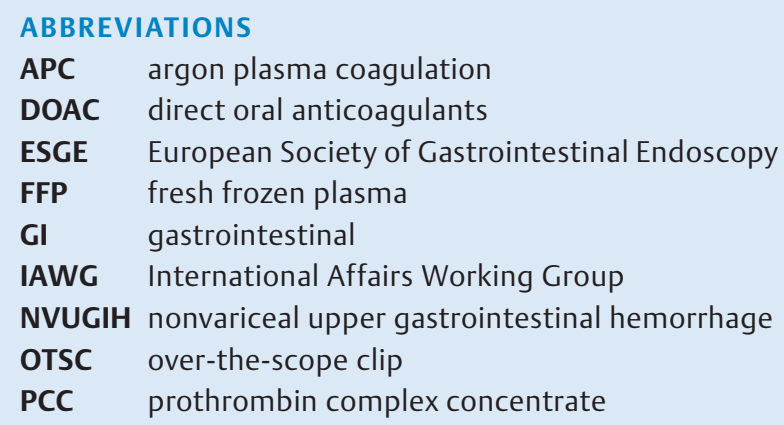

PPI proton pump inhibitor

TAE transcatheter angiographic embolization

UGIH upper gastrointestinal hemorrhage

VKA vitamin K antagonist

WEO World Endoscopy Organization 


\section{Introduction}

In order to address the status of gastrointestinal (GI) endoscopy in Africa, a European Society of Gastrointestinal Endoscopy (ESGE) International Affairs Working Group (IAWG) was established with participation from the World Endoscopy Organization (WEO) [1]. A previous survey conducted by the IAWG on the main indications of $\mathrm{Gl}$ endoscopy in African countries showed nonvariceal upper GI hemorrhage (NVUGIH) to be among the top three indications [1]. This indication may reflect the very high prevalence of Helicobacter pylori infection, which is well known to be associated with gastroduodenal peptic ulcers and their complications, such as bleeding. In addition, the increased use of aspirin as prevention and treatment of cardiovascular disease, and the increase in life expectancy, may also, at least in part, account for the NVUGIH indication [2 -4].

The management of NVUGIH requires a multi-step approach, involving endoscopic factors such as emergency endoscopy with different hemostatic devices, and clinical factors such as hospitalization, blood transfusion, and drugs. Such an approach is clearly sensitive to organizational, medical, pharmaceutical, and technological resources. However, the lack of structural endoscopic and clinical health resources in some underserved African areas is likely to affect the clinical outcome of patients with NVUGIH. In this regard, the previous IAWG survey revealed several critical needs in training, education, and technology that may represent a barrier to adequate NVUGIH management [1].

Most of the respondents from the African countries participating in the IAWG survey reported a lack of national guidelines for GI endoscopy; however, there was interest in the international guidelines, pending adaptation to reflect the endoscopic resources available in their regions. For this reason, the IAWG decided to apply the cascade methodology to adapt ESGE guidelines to resource-sensitive settings in partnership with African experts [1]. Cascade guidelines are resource oriented and provide hierarchical recommendations based on the re- sources available to the medical care provider [5,6]. In detail, four levels of resource availability-basic, limited, enhanced, and maximal levels-are identified, with most of the interest being in the basic and limited levels.

We aimed to standardize the management of NVUGIH in low-resources setting. Here, we report the cascade adaptation of the ESGE guideline on the diagnosis and management of NVUGIH [7].

\section{Methods}

Implementation of cascade methodology to ESGE guidelines has been detailed in a previous position paper [1]. Briefly, we selected resource-sensitive recommendations from the original ESGE guideline on NVUGIH [7]. Each recommendation was reviewed by four IAWG members. Those statements classified as resource sensitive by at least two reviewers were included in the revision process. The process of identification and selection of resource-sensitive statements from the original ESGE guideline, as well as their categorization into one of the four predefined resource levels, was conducted in close cooperation with four experts from Ghana and Nigeria. Subsequently, the IAWG, together with the first author of the original guideline, drafted a revision of the statements according to cascade methodology, for four predefined levels of resource availability ( $\downarrow$ Table 1 ) [8].

A modified Delphi process was then carried out with a panel of African gastroenterologists who were invited from a contact list of ESGE, WEO, and European national societies, as previously detailed $[1,8]$. If a $75 \%$ agreement was reached for all four levels of care (adaptations), the statement was accepted [8]. If the panel members disagreed with one of the adaptations, they had the opportunity to add a comment; thus, if an adaptation failed to reach agreement from $75 \%$ of the panel, the statement was revised according to the advice from the panel members. Subsequently, a second Delphi round might be conducted to reach an agreement on all of the resource-sensitive state-

- Table 1 Level of treatment based on resource availability.

\begin{tabular}{|c|c|}
\hline Predefined level & Definition \\
\hline $\begin{array}{l}\text { Basic } \\
\text { Levell }\end{array}$ & $\begin{array}{l}\text { Core resources or fundamental services absolutely necessary for an endoscopy care system to function. By definition, a health } \\
\text { care system lacking any basic level resource would be unable to provide endoscopic service to its patient population. It in- } \\
\text { cludes diagnostic procedures (gastroscopy and colonoscopy) as well and fundamental monitoring abilities (blood pressure, } \\
\text { basic blood biochemistry). }\end{array}$ \\
\hline $\begin{array}{l}\text { Limited } \\
\text { Level II }\end{array}$ & $\begin{array}{l}\text { Limited level: Second-tier resources or services that produce major improvements in outcome, such as increased survival, but } \\
\text { that are attainable with limited financial means and modest infrastructure. It includes minor endoscopic procedures to im- } \\
\text { prove major clinical outcomes (i. e. sclerotherapy/adrenaline injection, band ligation, plasma expanders, basic surgical in- } \\
\text { terventions). }\end{array}$ \\
\hline $\begin{array}{l}\text { Enhanced } \\
\text { Level III }\end{array}$ & $\begin{array}{l}\text { Enhanced level: Third-tier resources or services that are optional but important. Enhanced-level resources may produce mi- } \\
\text { nor improvements in outcome but increase the number and quality of therapeutic options. Most procedures that improves } \\
\text { clinical outcome are available (i. e. biliopancreatic endoscopy, electrosurgical unit, polypectomy/mucosectomy, anaesthesia } \\
\text { back-up). }\end{array}$ \\
\hline $\begin{array}{l}\text { Maximal } \\
\text { Level IV }\end{array}$ & $\begin{array}{l}\text { Maximal level: High-level resources or services that may be used in some high-resource countries or be recommended in } \\
\text { guidelines that assume unlimited resources. To be useful, maximal-level resources typically depend on the existence and } \\
\text { functionality of all lower-level resources. }\end{array}$ \\
\hline
\end{tabular}


Selection of resource-sensitive statements

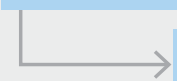

\section{Draft for adjustment of selected statements}

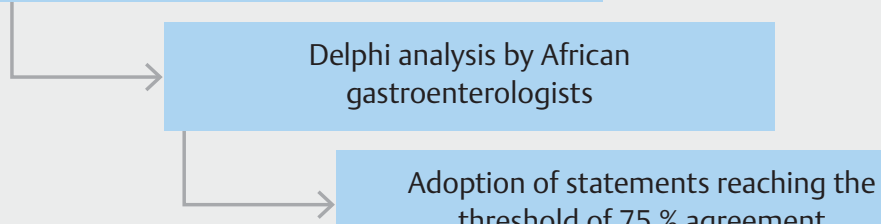
threshold of $75 \%$ agreement
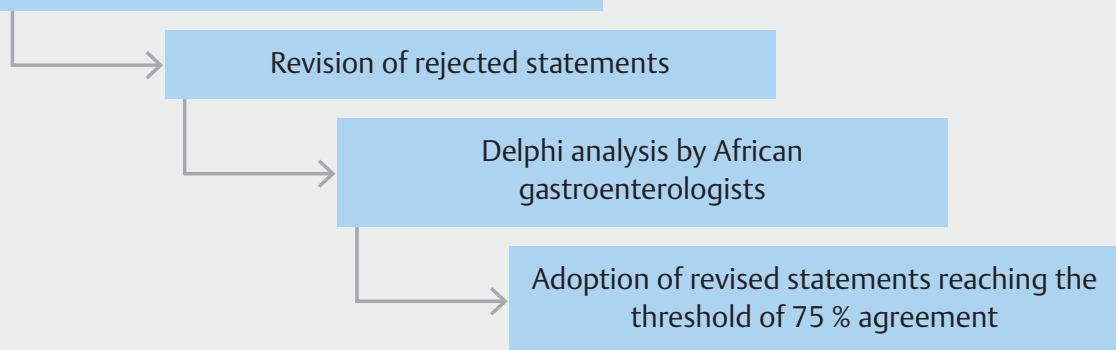

Fig. 1 Methodology of the Delphi process.

ments. Furthermore, if any panel member was not able to respond to specific statements during the Delphi process, they could refuse to answer. The methodology is summarized in - Fig. 1.

In order to describe background details of the Delphi panel, the participants were asked for the socioeconomic status of the location of their hospital and country, and which cascade level they were representing.

\section{Statement selection}

Of the 40 recommendations in the original ESGE guideline, 23 were selected as being resource sensitive by the IAWG. Four adapted cascade statements - one for each level - were created for each of the original recommendations, making a total of 92 adapted cascade guideline statements.

\section{The Delphi process}

Overall, 144 experts showed an interest in participating in the Delphi process, and 49 finally expressed their degree of agreement for one or more recommendations. Details of the 49 experts are shown in $>$ Table 2 .

A $\geq 75 \%$ agreement was achieved for $88 / 92$ proposed adaptations. Only four cascade adaptations of two recommendations failed to achieve the $\geq 75 \%$ agreement level. One of these failed adaptations was due to a misperceived lack of applicability of the Glasgow-Blatchford score to African settings. The other was a proposed adaptation to carry out endoscopy at the basic care level without INR in patients with coagulopathy due to vitamin antagonist. It was decided to omit the adaptation of these two recommendations.
- Table 2 Characteristics of the participants in the Delphi process.

\begin{tabular}{|l|c|}
\hline & $\begin{array}{c}\text { Participants, } \mathbf{n} \text { (\%) } \\
\text { (N=49) }\end{array}$ \\
\hline Geographical area, $\mathrm{n}(\%)$ & $27(55)$ \\
\hline - North Africa & $6(12)$ \\
\hline - Central Africa & $2(4)$ \\
\hline - East Africa & $14(29)$ \\
\hline - West Africa & $0(0)$ \\
\hline - Southern Africa & $3(6)$ \\
\hline Socioeconomic status of institution/hospital & \\
\hline - High & $26(53)$ \\
\hline - Mid & $20(41)$ \\
\hline - Low & \\
\hline Representation of cascade level 1 , 2 & $9(18)$ \\
\hline - Basic & $23(47)$ \\
\hline - Limited & $16(33)$ \\
\hline - Enhanced & $1(2)$ \\
\hline - Maximum & \\
\hline 1 Level determined and self-reported by Delphi panel experts. \\
\hline
\end{tabular}




\section{Cascade adaptation}

Each original recommendation with the accepted adaptations is reported in $>$ Table 3 . The main resources on which adaptation was performed may be categorized as follows.

1. Organizational - lack of availability of emergency endoscopy, radiology treatment, cardiologist consultancy, and anesthesiological assistance were reported as possible resource-sensitive limitations.

2. Endoscopy - lack of availability of epinephrine injection and second hemostatic modalities (i. e. thermal, clip, hemostatic spray) were reported as main barriers. In addition, endo- scopic or histological testing for $\mathrm{H}$. pylori was also considered to be resource sensitive.

3. Transfusions and drugs - transfusion strategies were affected at different levels by resource limitations. In detail, lack of biochemical assessment of hemoglobin levels hampers applicability of restrictive transfusion management. In addition, lack of transfusion of either blood, fresh frozen plasma or derivatives, as well as lack of crystalloids, may affect NVUGIH treatment. Proton pump inhibitor (PPI) availability may be limited as either intravenous or oral administration, and vitamin $\mathrm{K}$ may be lacking.

- Table 3 Adapted cascade recommendations for the management of nonvariceal upper gastrointestinal hemorrhage in resource-sensitive settings.

\section{Recommendation in original ESGE guideline ${ }^{1}$}

ESGE recommends a restrictive red blood cell transfusion strategy that aims for a target hemoglobin between $7 \mathrm{~g} / \mathrm{dL}$ and $9 \mathrm{~g} / \mathrm{dL}$. A higher target hemoglobin should be considered in patients with significant co-morbidity (e. g. ischemic cardiovascular disease) (strong recommendation, moderate quality evidence).

For patients taking vitamin $\mathrm{K}$ antagonists (VKAs), ESGE recommends withholding the VKA and correcting coagulopathy while taking into account the patient's cardiovascular risk in consultation with a cardiologist. In patients with hemodynamic instability, administration of vitamin K, supplemented with intravenous prothrombin complex concentrate (PCC) or fresh frozen plasma (FFP) if PCC is unavailable, is recommended (strong recommendation, low quality evidence).

ESGE recommends temporarily withholding new direct oral anticoagulants (DOACs) in patients with suspected acute NVUGIH in coordination/ consultation with the local hematologist/cardiologist (strong recommendation, very low quality evidence).

For patients using antiplatelet agents, ESGE recommends the management algorithm detailed in $\$$ Fig. 1 [ Fig. 2] (strong recommendation, moderate quality evidence).

ESGE recommends initiating high dose intravenous proton pump inhibitors (PPI), intravenous bolus followed by continuous infusion $(80 \mathrm{mg}$ then $8 \mathrm{mg} /$ hour), in patients presenting with acute UGIH awaiting upper endoscopy. However, PPI infusion should not delay the performance of early endoscopy (strong recommendation, high quality evidence).

In an effort to protect the patient's airway from potential aspiration of gastric contents, ESGE suggests endotracheal intubation prior to endoscopy in patients with ongoing active hematemesis, encephalopathy, or agitation (weak recommendation, low quality evidence).

ESGE recommends the availability of both an on-call GI endoscopist proficient in endoscopic hemostasis and on-call nursing staff with technical expertise in the use of endoscopic devices to allow performance of endoscopy on a $24 / 7$ basis (strong recommendation, moderate quality evidence).

\section{Adapted cascade recommendations}

Level I: Blood pressure monitoring and fluid resuscitation with crystalloid fluids

Level II: Restrictive blood transfusion strategy based on clinical judgment

Level III: No adjustment

Level I: Withhold VKA

Level II: Withhold VKA; correct coagulopathy with vitamin K after taking into account the patient's cardiovascular risk

Level III: Withhold VKA; correct coagulopathy with vitamin K after taking into account the patient's cardiovascular risk

Level I: Withhold DOACs

Level II: Withhold DOACs

Level III: Withhold DOACs + consultation with local hematologist and/or cardiologist

Level I: Saline or epinephrine injection; do not remove clot because second modality is not available; high dose oral or intravenous bolus PPI therapy; repeat endoscopy after $2-3$ days; empiric/pre-emptive $H$. pylori treatment; endoscopy together with surgeon; decision for surgery depends on various other factors

Level II: Epinephrine injection + second modality if available; do not remove clot if second treatment modality is not available; $H$. pylori testing and treatment when positive; consider $\mathrm{H}$. pylori treatment if one test is negative

Level III: No adjustment, but without topical hemostatic spray, over-thescope clip (OTSC) or transcatheter angiographic embolization (TAE)

Level I: Oral or intravenous bolus PPI

Level II: Intravenous bolus PPI

Level III: No adjustment

Level I: Patients with ongoing active hematemesis should be placed in a stable side position immediately; continuous active suction of blood and gastric contents

Level II: Stable side position; continuous sedation; continuous active suction of blood and gastric contents; emergency endoscopy Level III: No adjustment

Level I: Technical expertise may not be available on a 24/7 basis Level II: Endoscopy within 24 hours; trained emergency team with necessary technical expertise available

Level III: No adjustment 
- Table 3 (Continuation)

\section{Recommendation in original ESGE guideline ${ }^{1}$}

ESGE recommends that peptic ulcers with spurting or oozing bleeding (Forrest classification la and Ib, respectively) or with a nonbleeding visible vessel (Forrest classification Ila) receive endoscopic hemostasis because these lesions are at high risk for persistent bleeding or rebleeding (strong recommendation, high quality evidence).

ESGE recommends that peptic ulcers with an adherent clot (Forrest classification IIb) be considered for endoscopic clot removal. Once the clot is removed, any identified underlying active bleeding (Forrest classification la or lb) or nonbleeding visible vessel (Forrest classification Ila) should receive endoscopic hemostasis (weak recommendation, moderate quality evidence).

\section{Adapted cascade recommendations}

Level I: Endoscopic monotherapy; saline injection if no epinephrine; high dose PPI therapy; demonstration of endoscopy to a surgeon and together decide on optimal timing of surgery

Level II: Epinephrine injection + second treatment modality such as hemoclips or electrocoagulation; surgical consultation when necessary Level III: No adjustment, but without topical hemostatic spray, OTSC or TAE

Level I: Saline or epinephrine injection; do not remove clot because second modality is not available; high dose oral or intravenous bolus PPI therapy; repeat endoscopy after $2-3$ days; empiric/pre-emptive $H$. pylori treatment; endoscopy together with surgeon; decision for surgery depends on various other factors

Level II: Epinephrine injection + second modality if available; do not remove clot if second treatment modality is not available; $H$. pylori testing and treatment when positive; consider $\mathrm{H}$. pylori treatment if only one test was done and was negative; high dose oral or intravenous bolus PPI therapy

Level III: No adjustment, but without topical hemostatic spray, OTSC or TAE

Level I: Endoscopic monotherapy; saline injection if no epinephrine; high dose PPI therapy; demonstration of endoscopy to a surgeon and together decide on optimal timing of surgery. Also, consider endoscopic injection monotherapy using a sclerosing agent, e. g. alcohol, polidocanol, ethanolamine

Level II: Dilute epinephrine injection + second treatment modality such as hemoclips or electrocoagulation; surgical consultation when necessary

Level III: No adjustment

For patients with nonbleeding visible vessel (FIla), ESGE recommends mechanical therapy, thermal therapy, or injection of a sclerosing agent as monotherapy or in combination with epinephrine injection. ESGE recommends that epinephrine injection therapy not be used as endoscopic monotherapy (strong recommendation, high quality evidence).

For patients with active NVUGIH bleeding not controlled by standard endoscopic hemostasis therapies, ESGE suggests the use of a topical hemostatic spray or over-the-scope clip as salvage endoscopic therapy (weak recommendation, low quality evidence).

For patients with acid-related causes of NVUGIH different from peptic ulcers (e. g. erosive esophagitis, gastritis, duodenitis), ESGE recommends treatment with high dose PPI. Endoscopic hemostasis is usually not required and selected patients may be discharged early (strong recommendation, low quality evidence).

ESGE recommends that patients with a Mallory - Weiss lesion that is actively bleeding receive endoscopic hemostasis. There is currently inadequate evidence to recommend a specific endoscopic hemostasis modality. Patients with a Mallory - Weiss lesion and no active bleeding can receive high dose PPI therapy alone (strong recommendation, moderate quality evidence).
Level I: Endoscopic monotherapy (if available); saline injection if no epinephrine; high dose oral or intravenous bolus PPI therapy twice daily; consider repeat endoscopy after $2-3$ days; empiric/pre-emptive $H$. pylori treatment; endoscopy together with surgeon Level II: Endoscopic monotherapy (if available), saline injection if no epinephrine; second treatment modality (mechanical, contact thermal, sclerosing agent) if available; $H$. pylori testing and treatment when positive; however, consider $H$. pylori treatment if only one test was done and was negative; high dose oral or intravenous bolus PPI therapy twice daily Level III: No adjustment

Level I: Surgery

Level II: Surgery

Level III: Surgery or no adjustment

Level I: High dose oral PPI therapy twice daily

Level II and Level III: No adjustment

Level I: Injection therapy (or what is available); high dose PPI Level II: Injection therapy and/or second modality such as hemoclips or electrocoagulation (or what is available); high dose PPI Level III: No adjustment 
Table 3 (Continuation)

\section{Recommendation in original ESGE guideline ${ }^{1}$}

ESGE recommends that a Dieulafoy lesion receive endoscopic hemostasis using thermal, mechanical (hemoclip or band ligation), or combination therapy (dilute epinephrine injection combined with contact thermal or mechanical therapy) (strong recommendation, moderate quality evidence). Transcatheter angiographic embolization (TAE) or surgery should be considered if endoscopic treatment fails or is not technically feasible (strong recommendation, low quality evidence).

In patients bleeding from upper GI angioectasias, ESGE recommends endoscopic hemostasis therapy. However, there is currently inadequate evidence to recommend a specific endoscopic hemostasis modality (strong recommendation, low quality evidence).

In patients bleeding from upper GI neoplasia, ESGE recommends considering endoscopic hemostasis in order to avert urgent surgery and reduce blood transfusion requirements. However, no currently available endoscopic treatment appears to have long-term efficacy (weak recommendation, low quality evidence).

ESGE recommends PPI therapy for patients who receive endoscopic hemostasis and for patients with adherent clot not receiving endoscopic hemostasis. PPI therapy should be high dose and administered as an intravenous bolus followed by continuous infusion ( $80 \mathrm{mg}$ then $8 \mathrm{mg} /$ hour) for 72 hours post endoscopy (strong recommendation, high quality evidence).

ESGE suggests considering PPI therapy as intermittent intravenous bolus dosing (at least twice daily) for 72 hours post endoscopy for patients who receive endoscopic hemostasis and for patients with adherent clot not receiving endoscopic hemostasis. If the patient's condition permits, high dose oral PPI may also be an option in those able to tolerate oral medications (weak recommendation, moderate quality evidence).

In patients with clinical evidence of rebleeding following successful initial endoscopic hemostasis, ESGE recommends repeat upper endoscopy with hemostasis if indicated. In the case of failure of this second attempt at hemostasis, transcatheter angiographic embolization (TAE) or surgery should be considered (strong recommendation, high quality evidence).

In patients with NVUGIH secondary to peptic ulcer, ESGE recommends investigating for the presence of Helicobacter pylori in the acute setting with initiation of appropriate antibiotic therapy when $\mathrm{H}$. pylori is detected. Re-testing for $H$. pylori should be performed in those patients with a negative test in the acute setting. Documentation of successful $\mathrm{H}$. pylori eradication is recommended (strong recommendation, high quality evidence).

\section{Adapted cascade recommendations}

Level I: Injection therapy (or what is available); surgery if TAE unavailable Level II: Injection therapy and second treatment modality (mechanical, contact thermal) if available; surgery if TAE unavailable

Level III: No adjustment; surgery if TAE unavailable

Level I: Argon plasma coagulation (APC) or contact thermal therapy, e. g. bipolar or heat probe

Level II: APC or contact thermal therapy, e. g. bipolar or heat probe Level III: No adjustment

Level I: Endoscopic monotherapy (if available); saline injection if no epinephrine available

Level II: Endoscopic monotherapy (if available); saline injection if no epinephrine available; consider APC of tumor bleeding Level III: No adjustment; hemostatic spray if available

Level I: Intravenous bolus PPI twice daily instead of continuous infusion; if intravenous not available, use high dose oral PPI twice daily Level II and Level III: No adjustment

Level I: Intravenous PPI bolus twice daily instead of continuous infusion Level II and Level III: No adjustment

Level I: Endoscopic monotherapy; saline injection if no epinephrine is available; conventional histopathology for $\mathrm{H}$. pylori; empiric antibiotics for $\mathrm{H}$. pylori; intravenous PPI bolus if continuous infusion is impossible; surgery when TAE is unavailable

Level II: Endoscopic monotherapy; saline injection if no epinephrine is available; conventional histopathology for $\mathrm{H}$. pylori; empiric antibiotics for $H$. pylori; intravenous PPI bolus if continuous infusion is impossible; surgery when TAE is unavailable Level III: Surgery when TAE is unavailable

Level I: Conventional histopathology; consider empiric treatment when test is not available or if only one test was done and was negative Level II: Conventional histopathology and rapid urease test; consider empiric treatment if only one negative test was done Level III: No adjustment

ESGE, European Society of Gastrointestinal Endoscopy; GI, gastrointestinal; NVUGIH, nonvariceal upper gastrointestinal hemorrhage.

1 Gralnek IM, Dumonceau JM, Kuipers EJ et al. Diagnosis and management of nonvariceal upper gastrointestinal hemorrhage: European Society of Gastrointestinal Endoscopy (ESGE) Guideline. Endoscopy 2015; 47: a1 - 46

\section{Conclusions}

Our cascade approach was strengthened by the high rate of participating physicians practicing in low-to-mid socioeconomic areas, which corresponded closely with our definition of basic-to-limited settings of the cascade levels. Most of the original recommendations for NVUGIH management were successfully adapted to underserved areas, addressing limitations that mainly relate to infrastructural, pharmacological, and technological factors. For example, at the basic level, emergency endoscopy is not fully available, and pharmacological and surgical resources may therefore become more relevant. However, when emergency endoscopy is available, a lack of devices may reduce its efficacy; more affordable devices for a second thermal or mechanical hemostatic modality may then be more relevant for the outcome of these patients. 
High risk endoscopic stigmata identified (Fla, Flb, Flla, Fllb)

APA used for primary prophylaxis

- Withhold low dose acetylsalicylic acid (ASA)

- Re-evaluate risks and benefits of ongoing low dose ASA use

- Resume low dose ASA after ulcer healing or earlier if clinically indicated

APA used for secondary prophylaxis

(known cardiovascular disease)

1 Patients on low dose ASA alone

- Resume low dose ASA by day 3 following index endoscopy

- Second-look endoscopy at the discretion of the endoscopist may be considered

2 Patients on dual antiplatelet therapy (DAPT)

- Continue low dose ASA without interruption

- Early cardiology consultation for recommendation on resumption/continuation of second APA

- Second-look endoscopy at the discretion of the endoscopist may be considered
Low risk endoscopic stigmata identified (FIlc, FIII)

APA used for primary prophylaxis

- Withhold low dose ASA

- Re-evaluate risks and benefits of ongoing low dose ASA use

- Resume low dose ASA at hospital discharge if clinically indicated

APA used for secondary prophylaxis

(known cardiovascular disease)

1 Patients on low dose ASA alone

- Continue low dose ASA without interruption

2 Patients on dual antiplatelet therapy (DAPT)

- Continue DAPT without interruption

For patients using a non-ASA APA as monotherapy (e.g., thienopyridine alone), low-dose ASA may be given as substitute for interval period in patients with no contraindication or allergy to ASA.

Early cardiology consultation should be obtained for further APA recommendations.

Fig. 2 Algorithm for the management of patients with acute upper gastrointestinal hemorrhage who are using antiplatelet agent(s) [7].

\section{Disclaimer}

ESGE position statements and guidelines represent a consensus of best practice based on the available evidence at the time of preparation. They may not apply in all situations and should be interpreted in the light of specific clinical situations and resource availability. Further controlled clinical studies may be needed to clarify aspects of the statements, and revision may be necessary as new data appear. Clinical consideration may justify a course of action at variance to these recommendations. ESGE position statements and guidelines are intended to be an educational device to provide information that may assist endoscopists in providing care to patients. They are not rules and should not be construed as establishing a legal standard of care or as encouraging, advocating, requiring, or discouraging any particular treatment.

\section{Acknowledgments}

Participants of the Delphi panel: Benbouali Abdelhak Zaher, Benbouali Abdelhak Zaher, Halimi Abdelilah, Essaid Abdellah, Loumi Abderrahmane, Coulibaly Aboubacar, Amar Ahmed, Clausina Ahoui, Imen Akkari, Assia Alimohad, Sidahmed Karim
Allal, Gideon Anigbo, Tshimpi Antoine, Nourdin Aqodad, Mhamdia Asma, Abdou G. Ismael Assani, Constant Assi, Koffi Alai Attia, Mbarek Azouaoui, Nabil Ben Chaabane, Imed Ben Mansour, Aida Ben Slama, Azzedine Bendjaballah, Nassim Benhassine, Saadi Berkane, Bouhaouche Bilel, Mohamed Borahma, Riadh Bouali, Mona Boudabous, Alain Bougouma, Hanae Boutallaka, Joao Armindo Da Veiga, Nabil Debzi, Daouda Dia, Daouda Dia, Ibrahima Diallo, Titsaoui Djamila, Dr. Zanga Damien Ouattaram, Amine El Akkaoui, Fatima Zahra El Filali, Fatou Fall, Benas Faycal, Bossali Firmin, Alice Nanelin Guingane, Fouad Haddad, Daoudi Hadjer, Khaled Hallouly, Ziaya Hamdene, Lamine Hamzaoui, Uchenna Ijoma, Triki Ismail, Patrice Emery Itoudi Bignoumba, Hanen Jaziri, Nawal Kabbaj, Ghozlane Kacher, Doumbia Eps Samake Kadiatou, Rahal Kamel, Rostane Kamel, Dyane Kamto, Kadouche Khadidja, Abbas Khalida, Jellali Khaoula, Ghizlane Kharrasse, Amal Khsiba, Khadija Krati, Mohamed Hichem Loghmari, Wadhiha Louali, Bouchene Malik, Agnes Malongue, Gueye Mamadou Ngone, Charles Mbendi, Hailemichael Mekonnen, Yunus Miya, Hameche Moad, Ndiaye Mohamed Fadel, El Yamani Mohamed Lyamani, Belkahla Mohamed Reda, Ainsebaa Mohammed, Amrani Mohammed, Benmeryeme Mohammed, Marref Mohammed, Monique Esther Mbounja Ep Zue Ndoutoumou, Diarra Moussa, Jean Baptiste 
Moussavou Kombila, Dafiri Najia, Delphine Napon-Zongo, Rénovat Ntagirabiri, Sylvester Nwokediuko, Chegdali Ouafae, Sofia Oubaha, Ali Oukaour, Mbaye Papa Saliou, Talla Paul, Meriam Sabbah, Sabrina Sahri, Nacer Saib, El Jastimi Said, Mouna Salihoun, Tafat Salim, Rodrigue Salou, Boualam Samira, Zouhour Samlani, Addou Sidi Mohamed, Oulidali Sohaib, Roger Sombie, Amel Tata, Dorra Trad, Bouhlel Wided, Aissat Yakhlef, Mejda Zakhama, Hailemichael Desalegn, Berhane Redae.

\section{Competing interests}

None

\section{References}

[1] Hassan C, Aabakken L, Ebigbo A et al. Partnership with African countries: European Society of Gastrointestinal Endoscopy (ESGE) Position Statement. Endosc Int Open 2018; 06: E1247 -E1255
[2] Hellmig S, Hampe J, Schreiber S. Helicobacter pylori infection in Africa and Europe: enigma of host genetics. Gut 2003; 52: 1799

[3] Tanih NF, Dube C, Green E et al. An African perspective on Helicobacter pylori: prevalence of human infection, drug resistance, and alternative approaches to treatment. Ann Trop Med Parasitol 2009; 103: 189 204

[4] Malfertheiner P, Chan FK, McColl KE. Peptic ulcer disease. Lancet 2009; 374: 1449-1461

[5] Fried M, Krabshuis J. Can ‘Cascades' make guidelines global? J Eval Clin Pract 2008; 14: 874 - 879

[6] Malagelada JR, Bazzoli F, Boeckxstaens G et al. World Gastroenterology Organisation Global Guidelines: Dysphagia - global guidelines and cascades update September 2014. J Clin Gastroenterol 2015; 49: $370-378$

[7] Gralnek IM, Dumonceau JM, Kuipers EJ et al. Diagnosis and management of nonvariceal upper gastrointestinal hemorrhage: European Society of Gastrointestinal Endoscopy (ESGE) Guideline. Endoscopy 2015; 47: a $1-46$

[8] Milholland AV, Wheeler SG, Heieck JJ. Medical assessment by a Delphi group opinion technic. N Engl J Med 1973; 288: 1272 - 1275 